\title{
The one dimensional parabolic $p(x)$-Laplace equation
}

\author{
Alkis S. Tersenov
}

\begin{abstract}
The Dirichlet problem for the degenerate and singular parabolic $p(x)$-Laplace equation with one spatial variable is considered. We prove the existence of the unique weak solution such that the derivatives $u_{t}$ and $u_{x}$ of a solution $u$ belong to $L_{\infty}$. Moreover for the singular case we prove the existence of the strong solution i.e. such that $u_{t}, u_{x}$ and $u_{x x}$ belong to $L_{\infty}$.
\end{abstract}

Mathematics Subject Classification. 35K20, 35K65, 35B45.

Keywords. Singular parabolic equation, Degenerate parabolic equation.

\section{Introduction and formulation of the results}

Consider the following quasilinear parabolic equation

$$
u_{t}=\left(\left|u_{x}\right|^{p(x)} u_{x}\right)_{x}+f\left(x, u, u_{x}\right) \quad \text { in } Q_{T}=(0, T) \times(-l, l),
$$

coupled with the initial and homogeneous Dirichlet boundary conditions

$$
u(0, x)=u_{0}(x) \text { for }|x| \leq l \text { and } u(t, \pm l)=0 \text { for } t \in[0, T]
$$

where

$$
\max _{|x| \leq l}\left|\left(\left|u_{0 x}\right|^{p(x)} u_{0 x}\right)_{x}\right|<\infty \quad \text { and } \quad u_{0}( \pm l)=0 .
$$

Here $T, l$ are arbitrary positive constants and the function $p(x)>-1$ for $x \in[-l, l]$

The case when $p$ is constant was studied by a lot of authors and optimal results concerning this equation was obtained (see, for example, [5]). There recently appeared a large number of publications with non constant $p$, see [1$4,6,8,9,12]$ and the references therein. In [8] the multidimensional case was considered and it was proved that if $p(x)>0$ is a measurable function, $f=$ $f(t, x, u)$ is $C^{1}$ function and $u_{0} \in L^{\infty} \cap W_{0}^{1, p(x)}$, then there exists a global bounded weak solution of the problem such that

$$
u \in L^{\infty}\left(0, T ; W_{0}^{1, p(x)}\right), \quad u_{t} \in L^{2} .
$$


For more details see [8]. Our goal in the present paper is to obtain the global solution with essentially better differential properties in the one dimensional case. To this end we need $p(x)$ to be $C^{1}$ function, but in contrast with [8] we consider the singular case as well (i.e. $-1<p(x) \leq 0$ ). Concerning the function $f$, first we assume that $f=f\left(x, u, u_{x}\right)$ and second we do not need $f$ to be $C^{1}$ function but $C^{\gamma}$, with $\gamma \in(0,1)$. Concerning the assumption on $u_{0}$ see (1.3). We show that the derivatives $u_{t}$ and $u_{x}$ are $L^{\infty}$ functions, moreover if $-1<p(x) \leq 0$ then $u_{x x}$ belongs to $L^{\infty}$ as well.

Assume that

$$
u f(x, u, 0) \leq \alpha u^{2}+\beta,
$$

here $\alpha$ and $\beta$ are some nonnegative constants,

$$
|f(x, u, q)| \leq|q|^{p(x)} \psi(|q|) \quad \text { for } \quad|x| \leq l, \quad|u| \leq M,
$$

the constant $M$ will be defined below (see (2.3)), the function $\psi$ is smooth, nonnegative, nondecreasing function such that

$$
\begin{gathered}
\int^{\infty} \frac{\rho d \rho}{\psi(\rho)}=+\infty, \\
f\left(x, u_{2}, q\right)-f\left(x, u_{1}, q\right) \leq 0 \quad \text { for } u_{2}>u_{1}, \\
p(x) \in C^{1}([-l, l]), \quad f(x, u, q) \in C^{\gamma}\left([-l, l] \times \mathbf{R}^{2}\right), \quad \gamma \in(0,1) .
\end{gathered}
$$

Definition 1. We say that a Lipschitz continuous function $u(t, x): Q_{T} \rightarrow \mathbf{R}$ is a strong solution of problem (1.1), (1.2) if $u_{x x} \in L_{\infty}\left(Q_{T}\right)$ and the equation

$$
u_{t}=(1+p(x))\left|u_{x}\right|^{p(x)} u_{x x}+p^{\prime}(x) u_{x}\left|u_{x}\right|^{p(x)} \ln \left|u_{x}\right|+f\left(x, u, u_{x}\right)
$$

is satisfied almost everywhere in $Q_{T}$. Initial and boundary conditions are satisfied in the classical sense.

We put

$$
b(x, 0)=0 \quad \text { for } \quad b(x, z) \equiv p^{\prime}(x) z|z|^{p(x)} \ln |z| .
$$

Theorem 1. Assume that conditions (1.3)-(1.7) are fulfilled. If $p(x) \in(-1,0]$ for $x \in[-l, l]$, then for an arbitrary $T>0$, there exists a strong solution of problem (1.1), (1.2). unique.

If, in addition, $f$ is Lipschitz continuous function, then the solution is

Definition 2. We say that a Lipschitz continuous function $u(t, x): Q_{T} \rightarrow \mathbf{R}$ is a weak solution of problem (1.1), (1.2) if it satisfies the following integral identity

$$
\int_{Q_{T}}\left(u_{t} \phi+\left|u_{x}\right|^{p(x)} u_{x} \phi_{x}\right) d t d x=\int_{Q_{T}} f\left(x, u, u_{x}\right) \phi d t d x
$$

for an arbitrary smooth function $\phi(t, x)$ such that $\phi(t, \pm l)=0$ on $(0, T)$. Initial and boundary condition are satisfied in the classical sense. 
In order to formulate the next theorem we need to substitute condition (1.5) by more restrictive one, namely, we suppose that $f$ is linear with respect to $u_{x}$ i.e.

$$
f\left(x, u, u_{x}\right)=g_{1}(x, u) u_{x}+g_{2}(x, u) .
$$

Theorem 2. Assume that conditions (1.3), (1.4), (1.6)-(1.8) are fulfilled. Then for an arbitrary $T>0$, there exists a weak solution $u$ of problem (1.1), (1.2). unique.

If, in addition, $f$ is Lipschitz continuous function, then the solution is

\section{A priori estimates for the regularized problem}

\subsection{Regularization}

Consider the regularized equation

$$
u_{\varepsilon t}=\left(\left(u_{\varepsilon x}^{\alpha}+\varepsilon\right)^{\frac{p(x)}{\alpha}} u_{\varepsilon x}\right)_{x}+f\left(x, u_{\varepsilon}, u_{\varepsilon x}\right) .
$$

Rewrite this equation in the equivalent form

$$
u_{\varepsilon t}=a\left(\varepsilon, x, u_{\varepsilon x}\right) u_{\varepsilon x x}+b\left(\varepsilon, x, u_{\varepsilon x}\right)+f\left(x, u_{\varepsilon}, u_{\varepsilon x}\right) .
$$

where

$$
a\left(\varepsilon, x, u_{\varepsilon x}\right)=\left(u_{\varepsilon x}^{\alpha}+\varepsilon\right)^{\frac{p(x)}{\alpha}}\left(1+p(x) \frac{u_{\varepsilon x}^{\alpha}}{u_{\varepsilon x}^{\alpha}+\varepsilon}\right)
$$

and

$$
b\left(\varepsilon, x, u_{\varepsilon x}\right)=p^{\prime}(x) u_{\varepsilon x}\left(u_{\varepsilon x}^{\alpha}+\varepsilon\right)^{\frac{p(x)}{\alpha}} \ln \left(u_{\varepsilon x}^{\alpha}+\varepsilon\right)^{\frac{1}{\alpha}} .
$$

Here constants $\varepsilon$ and $\alpha$ belong to $(0,1)$.

We additionally suppose that $\alpha=r / m$ with positive integers $r$ and $m$ such that $r<m$ and $m$ is even. For such $\alpha$

$$
z^{\alpha}=|z|^{\alpha} \quad \text { and } \quad\left(z^{\alpha}\right)^{p / \alpha}=|z|^{p} .
$$

The existence of a classical solution $u_{\varepsilon}$ of problem (2.1), (1.2), follows from [11].

\subsection{A priori estimates}

Our goal in this section is to obtain uniform with respect to $\varepsilon$ estimates of this solution which would enable us to pass to the limit as $\varepsilon \rightarrow 0$.

First we mention that (1.4) implies the estimate

$$
\left|u_{\varepsilon}(t, x)\right| \leq M=\inf _{\lambda>\alpha} e^{\lambda T}\left[\max _{\Gamma_{T}}|u|,\left(\frac{\beta}{\lambda-\alpha}\right)^{1 / 2}\right],
$$

for every $\varepsilon \in(0,1)$ (see $[7$, relation $(2.31)]$ ). By $\Gamma_{T}$ we denote the parabolic boundary of $Q_{T}$ i.e.

$$
\Gamma_{T}=\partial \bar{Q}_{T} \backslash\{t=T,|x|<l\} .
$$


Denote by $K$ the following quantity:

$$
K=\max _{x, \varepsilon}\left|\left(\left(u_{0 x}^{\alpha}+\varepsilon\right)^{\frac{p(x)}{\alpha}} u_{0 x}\right)_{x}\right|+\max _{x, u}\left|f\left(x, u, u_{0 x}\right)\right|<\infty,
$$

here maximum is taking over the set $x \in[-l, l], \varepsilon \in[0,1], u \in[-M, M]$.

We start with the estimate of $u_{\varepsilon t}$ at $t=0$.

Lemma 2.1. For every $\varepsilon \in(0,1)$ the following inequality

$$
\left|u_{\varepsilon}(t, x)-u_{0}(x)\right| \leq K t, \quad \forall(t, x) \in Q_{T}
$$

takes place.

Proof. For simplicity we will omit in the proof the subindex $\varepsilon$.

Introduce the function

$$
h(t)=(K+\delta) t \quad \text { in } \quad[0, T],
$$

where $\delta>0$. Let us prove the following inequality

$$
u(t, x)-u_{0}(x) \leq h(t) \quad \text { for } \quad(t, x) \in \bar{Q}_{T} .
$$

Consider the linear operator

$$
L \equiv \frac{\partial}{\partial x}\left(\left(\left|u_{0 x}\right|^{\alpha}+\varepsilon\right)^{p(x) / \alpha} \frac{\partial}{\partial x}\right)-\frac{\partial}{\partial t} .
$$

Define the function $\phi^{+}(t, x) \equiv u(t, x)-\left[u_{0}(x)+h(t)\right]$, obviously

$$
\begin{aligned}
L \phi^{+}= & \frac{\partial}{\partial x}\left(\left(\left|u_{0 x}\right|^{\alpha}+\varepsilon\right)^{p(x) / \alpha} \frac{\partial u}{\partial x}\right)-\frac{\partial u}{\partial t} \\
& -\frac{\partial}{\partial x}\left(\left(\left|u_{0 x}\right|^{\alpha}+\varepsilon\right)^{p(x) / \alpha} \frac{\partial u_{0}}{\partial x}\right)+K+\delta \\
> & \left(\left(\left|u_{0 x}\right|^{\alpha}+\varepsilon\right)^{p(x) / \alpha} u_{x}\right)_{x}-u_{t}+\left|f\left(x, u, u_{0 x}\right)\right| .
\end{aligned}
$$

Suppose that at some point $N \in \bar{Q}_{T} \backslash \Gamma_{T}$ the function $\phi^{+}$attains its maximum, then at this point

$$
\phi_{x}^{+}=0 \quad \Leftrightarrow \quad u_{x}=u_{0 x}
$$

and hence

$$
\begin{aligned}
& \left(\left(\left|u_{0 x}\right|^{\alpha}+\varepsilon\right)^{p(x) / \alpha} u_{x}\right)_{x}-u_{t}+\left.\left|f\left(x, u, u_{0 x}\right)\right|\right|_{N} \\
& \quad=\left(\left(\left|u_{x}\right|^{\alpha}+\varepsilon\right)^{p(x) / \alpha} u_{x}\right)_{x}-u_{t}+\left.\left|f\left(x, u, u_{x}\right)\right|\right|_{N} \\
& \quad=-f\left(x, u, u_{x}\right)+\left.\left|f\left(x, u, u_{x}\right)\right|\right|_{N} \geq 0 .
\end{aligned}
$$

Thus, from (2.5)

$$
\left.L \phi^{+}\right|_{N}>0
$$

which contradicts the assumption that $\phi^{+}$attains its maximum at $N$.

Consider $\phi^{+}$on $\Gamma_{T}$ :

for $x= \pm l, t \in[0, T]$ we have $\phi^{+}=-h(t) \leq 0$;

for $t=0,|x| \leq l$ we have $\phi^{+}=-h(0)=0$. 
Thus $\phi^{+} \leq 0$ on $\Gamma_{T}$ and consequently

$$
\phi^{+} \leq 0 \quad \text { in } \quad \bar{Q}_{T} .
$$

Inequality (2.4) is proved.

Let us show now that

$$
u(t, x)-u_{0}(x) \geq-h(t) \quad \text { for } \quad(t, x) \in \bar{Q}_{T} .
$$

For the function $\phi^{-}(t, x) \equiv u(t, x)-\left[u_{0}(x)-h(t)\right]$ we have

$$
\begin{aligned}
L \phi^{-} & =\left(\left(\left|u_{0 x}\right|^{\alpha}+\varepsilon\right)^{p(x) / \alpha} u_{x}\right)_{x}-u_{t}-\left(\left(\left|u_{0 x}\right|^{\alpha}+\varepsilon\right)^{p(x) / \alpha} u_{0 x}\right)_{x}-K-\delta \\
& <\left(\left(\left|u_{0 x}\right|^{\alpha}+\varepsilon\right)^{p(x) / \alpha} u_{x}\right)_{x}-u_{t}-\left|f\left(x, u, u_{0 x}\right)\right| .
\end{aligned}
$$

Suppose that at some point $N_{1} \in \bar{Q}_{T} \backslash \Gamma_{T}$ the function $\phi^{-}$attains its minimum, then at this point

$$
\phi_{x}^{-}=0 \quad \Leftrightarrow \quad u_{x}=u_{0 x}
$$

and

$$
\begin{aligned}
& \left(\left(\left|u_{0 x}\right|^{\alpha}+\varepsilon\right)^{p(x) / \alpha} u_{x}\right)_{x}-u_{t}-\left.\left|f\left(x, u, u_{0 x}\right)\right|\right|_{N_{1}} \\
& =\left(\left(\left|u_{x}\right|^{\alpha}+\varepsilon\right)^{p / \alpha} u_{x}\right)_{x}-u_{t}-\left.\left|f\left(x, u, u_{x}\right)\right|\right|_{N_{1}} \\
& =-f\left(x, u, u_{x}\right)-\left.\left|f\left(x, u, u_{x}\right)\right|\right|_{N_{1}} \leq 0,
\end{aligned}
$$

hence, from $(2.7)$

$$
\left.L \phi^{-}\right|_{N_{1}}<0
$$

which contradicts the assumption that $\phi^{-}$attains its minimum at $N_{1}$. On $\Gamma_{T}$ we have $\phi^{-} \geq 0$, hence

$$
\phi^{-} \geq 0 \quad \text { in } \quad \bar{Q}_{T}
$$

and (2.6) is proved. From (2.4) and (2.6) we obtain that

$$
\left|u(t, x)-u_{0}(x)\right| \leq h(t) \quad \text { in } \quad \bar{Q}_{T} .
$$

Passing to the limit when $\delta \rightarrow 0$ we finish the prove of Lemma 2.1.

We turn now to the global estimate of the time derivative.

Lemma 2.2. For every $\varepsilon \in(0,1)$ the following estimate

$$
\left|u_{\varepsilon t}\right| \leq K, \quad \forall(t, x) \in Q_{T},
$$

takes place.

Proof. As in the prove of the previous lemmas, here we also omit the subindex $\varepsilon$.

Consider Eq. (2.2) in two different points:

$$
u_{t}(t, x)=a\left(\varepsilon, x, u_{x}(t, x)\right) u_{x x}(t, x)+b\left(\varepsilon, x, u_{x}(t, x)\right)+f\left(x, u(t, x), u_{x}(t, x)\right)
$$


and

$u_{\tau}(\tau, x)=a\left(\varepsilon, x, u_{x}(\tau, x)\right) u_{x x}(\tau, x)+b\left(\varepsilon, x, u_{x}(\tau, x)\right)+f\left(x, u(\tau, x), u_{x}(\tau, x)\right)$

where $t \neq \tau$. we have

Subtracting (2.9) from (2.8) for the function $v(t, \tau, x) \equiv u(t, x)-u(\tau, x)$

$$
\begin{aligned}
v_{t}+ & v_{\tau}-a\left(\varepsilon, x, u_{x}(t, x)\right) v_{x x} \\
= & {\left[a\left(\varepsilon, x, u_{x}(t, x)\right)-a\left(\varepsilon, x, u_{x}(\tau, x)\right)\right] u_{x x}(\tau, x) } \\
& +\left[b\left(\varepsilon, x, u_{x}(t, x)\right)-b\left(\varepsilon, x, u_{x}(\tau, x)\right)\right] \\
& +\left[f\left(x, u(t, x), u_{x}(t, x)\right)-f\left(x, u(\tau, x), u_{x}(\tau, x)\right)\right] .
\end{aligned}
$$

To obtain the above equation we use the following obvious relations

$$
\begin{aligned}
v_{t}(t, \tau, x) & =u_{t}(t, x), \quad v_{\tau}(t, \tau, x)=-u_{\tau}(\tau, x), \\
v_{x x}(t, \tau, x) & =u_{x x}(t, x)-u_{x x}(\tau, x),
\end{aligned}
$$

Define the function

$$
\mathrm{w} \equiv v-K(t-\tau)=u(t, x)-u(\tau, x)-K(t-\tau)
$$

in the domain

$$
P=\{(t, \tau, x): t \in(0, T), \tau \in(0, T),|x|<l, t>\tau\} .
$$

The function w satisfies the following relation:

$$
\begin{aligned}
\mathrm{w}_{t}+ & \mathrm{w}_{\tau}-a\left(\varepsilon, x, u_{x}(t, x)\right) \mathrm{w}_{x x} \\
= & {\left[a\left(\varepsilon, x, u_{x}(t, x)\right)-a\left(\varepsilon, x, u_{x}(\tau, x)\right)\right] u_{x x}(\tau, x) } \\
& +\left[b\left(\varepsilon, x, u_{x}(t, x)\right)-b\left(\varepsilon, x, u_{x}(\tau, x)\right)\right] \\
& +\left[f\left(x, u(t, x), u_{x}(t, x)\right)-f\left(x, u(\tau, x), u_{x}(\tau, x)\right)\right] .
\end{aligned}
$$

Introduce the function

$$
\omega \equiv \mathrm{w} e^{-\tau}
$$

which satisfies in $P$ the following linear ultraparabolic equation

$$
\begin{aligned}
L \omega \equiv & \omega_{t}+\omega_{\tau}+\omega-a\left(\varepsilon, x, u_{x}(t, x)\right) \omega_{x x} \\
= & e^{-\tau}\left(\left[a\left(\varepsilon, x, u_{x}(t, x)\right)-a\left(\varepsilon, x, u_{x}(\tau, x)\right)\right] u_{x x}(\tau, x)\right. \\
& \left.+\left[b\left(\varepsilon, x, u_{x}(t, x)\right)-b\left(\varepsilon, x, u_{x}(\tau, x)\right)\right]\right) \\
& +e^{-\tau}\left(\left[f\left(x, u(t, x), u_{x}(t, x)\right)-f\left(x, u(\tau, x), u_{x}(\tau, x)\right)\right]\right) .
\end{aligned}
$$

Let

$$
\Gamma_{\tau}=\partial P \backslash\{(t, \tau, x): t=T, 0<\tau<T,|x|<l\} .
$$

Suppose that the function $\omega$ attains its positive maximum at some point $N\left(t_{0}, \tau_{0}, x_{0}\right) \in \bar{P} \backslash \Gamma_{\tau}$. At this point it should be

$$
\left.L \omega\right|_{N}>0,
$$


since

$$
\omega_{t}(N) \geq 0, \quad \omega_{\tau}(N) \geq 0, \quad \omega(N)>0 \text { and }-\omega_{x x}(N) \geq 0 .
$$

On the other hand at this point $\omega_{x}=0$ i.e.

$$
u_{x}\left(t_{0}, x_{0}\right)=u_{x}\left(\tau_{0}, x_{0}\right),
$$

hence

$$
\begin{aligned}
& a\left(\varepsilon, x_{0}, u_{x}\left(t_{0}, x_{0}\right)\right)=a\left(\varepsilon, x_{0}, u_{x}\left(\tau_{0}, x_{0}\right)\right), \\
& b\left(\varepsilon, x_{0}, u_{x}\left(t_{0}, x_{0}\right)\right)=b\left(\varepsilon, x_{0}, u_{x}\left(\tau_{0}, x_{0}\right)\right)
\end{aligned}
$$

and, since $u\left(t_{0}, x_{0}\right)>u\left(\tau_{0}, x_{0}\right)$,

$$
f\left(x_{0}, u\left(t_{0}, x_{0}\right), u_{x}\left(t_{0}, x_{0}\right)\right) \leq f\left(x_{0}, u\left(\tau_{0}, x_{0}\right), u_{x}\left(\tau_{0}, x_{0}\right)\right),
$$

the last is due to condition (1.6). Thus (2.11) implies that

$$
\left.L \omega\right|_{N} \leq 0 \text {. }
$$

From this contradiction we conclude that $\omega$ can not attain its positive maximum in $\bar{P} \backslash \Gamma_{\tau}$.

Consider $\omega$ on $\Gamma_{\tau}$ :

for $|x|=l, t \in[0, T], \tau \in[0, T]$ we have $\omega=-K(t-\tau) e^{-\tau} \leq 0$;

for $t=\tau,|x|<l, t \in[0, T]$ we have $\omega=0$;

for $\tau=0, t \in[0, T],|x|<l$ we have $\omega=u(t, x)-u_{0}(x)-K t \leq 0$ due to Lemma 2.1.

Consequently $\omega \leq 0$ in $\bar{P}$ i.e.

$$
u(t, x)-u(\tau, x) \leq K(t-\tau) .
$$

Now subtracting (2.8) from (2.9) for the function $\tilde{v}(t, \tau, x) \equiv u(\tau, x)-$ $u(t, x)$ we obtain

$$
\begin{aligned}
\tilde{v}_{t}+ & \tilde{v}_{\tau}-a\left(\varepsilon, x, u_{x}(\tau, x)\right) \tilde{v}_{x x} \\
= & {\left[a\left(\varepsilon, x, u_{x}(\tau, x)\right)-a\left(\varepsilon, x, u_{x}(t, x)\right)\right] u_{x x}(t, x) } \\
& +\left[b\left(\varepsilon, x, u_{x}(\tau, x)\right)-b\left(\varepsilon, x, u_{x}(t, x)\right)\right] \\
& +\left[f\left(x, u(\tau, x), u_{x}(\tau, x)\right)-f\left(x, u(t, x), u_{x}(t, x)\right)\right] .
\end{aligned}
$$

Define the function

$$
\tilde{\mathrm{w}} \equiv \tilde{v}-K(t-\tau)=u(\tau, x)-u(t, x)-K(t-\tau),
$$

which satisfies in $P$ the following relation:

$$
\begin{aligned}
\tilde{\mathrm{w}}_{t}+ & \tilde{\mathrm{w}}_{\tau}-a\left(\varepsilon, x, u_{x}(\tau, x)\right) \tilde{\mathrm{w}}_{x x} \\
= & {\left[a\left(\varepsilon, x, u_{x}(\tau, x)\right)-a\left(\varepsilon, x, u_{x}(t, x)\right)\right] u_{x x}(t, x) } \\
& +\left[b\left(\varepsilon, x, u_{x}(\tau, x)\right)-b\left(\varepsilon, x, u_{x}(t, x)\right)\right] \\
& +\left[f\left(x, u(\tau, x), u_{x}(\tau, x)\right)-f\left(x, u(t, x), u_{x}(t, x)\right)\right] .
\end{aligned}
$$

Introduce the function

$$
\tilde{\omega} \equiv \tilde{\mathrm{w}} e^{-\tau}
$$


which satisfies in $P$ the following linear ultraparabolic equation

$$
\begin{aligned}
L \tilde{\omega} \equiv \tilde{\omega}_{t} & +\tilde{\omega}_{\tau}+\tilde{\omega}-a\left(\varepsilon, x, u_{x}(\tau, x)\right) \tilde{\omega}_{x x} \\
=e^{-\tau} & \left(\left[a\left(\varepsilon, x, u_{x}(\tau, x)\right)-a\left(\varepsilon, x, u_{x}(t, x)\right)\right] u_{x x}(t, x)\right] \\
& \left.+\left[b\left(\varepsilon, x, u_{x}(\tau, x)\right)-b\left(\varepsilon, x, u_{x}(t, x)\right)\right]\right) \\
& +e^{-\tau}\left(\left[f\left(x, u(\tau, x), u_{x}(\tau, x)\right)-f\left(x, u(t, x), u_{x}(t, x)\right)\right]\right) .
\end{aligned}
$$

Similarly to the previous case we obtain that $\tilde{\omega}$ can not attain its positive maximum in $\bar{P} \backslash \Gamma_{\tau}$ and that $\tilde{\omega} \leq 0$ on $\Gamma_{\tau}$.

Consequently $\tilde{\omega} \leq 0$ in $\bar{P}$ i.e.

$$
u(\tau, x)-u(t, x) \leq K(t-\tau) .
$$

From (2.12) and (2.12) we conclude that in $\bar{P}$

$$
|u(t, x)-u(\tau, x)| \leq K(t-\tau) .
$$

Taking into account the symmetry of the variables $t$ and $\tau$ we similarly consider the case $t<\tau$ to obtain that in

$$
\{(t, \tau, x): t \in[0, T], \tau \in[0, T], x \in[-l, l]
$$

the inequality

$$
|u(t, x)-u(\tau, x)| \leq K|t-\tau|
$$

holds. The last implies the required estimate.

We also need the estimates of the spatial derivative of the solution.

Lemma 2.3. There exists a constant $C_{0}$ independent of $\varepsilon$ such that

$$
\left|u_{\varepsilon x}\right| \leq C_{0},
$$

for every $\varepsilon \in(0,1)$.

Proof. This lemma follows from [11]. In fact, taking into account (1.5) we see that there exists a smooth, nonnegative, nondecreasing function $\bar{\psi}$ such that

$$
\int^{\infty} \frac{\rho d \rho}{\bar{\psi}(\rho)}=+\infty
$$

and

$$
\left|f\left(x, u_{\varepsilon}, q\right)+b(\varepsilon, x, q)\right| \leq a(\varepsilon, x, q) \bar{\psi}(|q|) \quad \forall \varepsilon \geq 0 .
$$

Thus (see Lemma 3 from [11]) the estimate $\left|u_{\varepsilon x}\right| \leq C_{0}$ is true with $C_{0}$ depending only on $M$ and $\bar{\psi}$.

Let us obtain the estimate of the second derivative for the singular case.

Lemma 2.4. Suppose that $-1<p(x) \leq 0$ for $x \in[-l, l]$. Then, there exists a constant $C_{1}$ independent of $\varepsilon$ such that for every $\varepsilon \in(0,1)$ the following estimate takes place

$$
\left|u_{\varepsilon x x}\right| \leq C_{1}
$$


Proof. From (2.2) we have (we omit the subindex $\varepsilon$ ):

$$
\left|u_{x x}\right| \leq \frac{\left|u_{t}\right|}{a}+\frac{|b|}{a}+\frac{|f|}{a} .
$$

In order to obtain the needed estimate (taking into account the estimates obtained in the previous lemmas) it is sufficient to estimate the term $a\left(\varepsilon, x, u_{x}\right)$ from the below uniformly with respect to $\varepsilon$. We have

$$
\left(u_{x}^{\alpha}+\varepsilon\right)^{\frac{p(x)}{\alpha}} \geq\left(C_{0}^{\alpha}+1\right)^{\frac{p(x)}{\alpha}}
$$

taking into account that

$$
0<1+p^{-} \leq 1+p(x) \frac{u_{x}^{\alpha}}{u_{x}^{\alpha}+\varepsilon}, \quad \text { where } \quad p^{-}=\min _{|x| \leq l} p(x)>-1,
$$

we obtain

$$
a\left(\varepsilon, x, u_{x}\right)=\left(u_{x}^{\alpha}+\varepsilon\right)^{\frac{p(x)}{\alpha}}\left(1+p(x) \frac{u_{x}^{\alpha}}{u_{x}^{\alpha}+\varepsilon}\right) \geq\left(C_{0}^{\alpha}+1\right)^{\frac{p(x)}{\alpha}}\left(1+p^{-}\right) .
$$

Note that both in Lemmas 2.3 and 2.4 we essentially use that we have only one spatial variable.

\section{Proof of Theorems 1 and 2}

We will obtain a strong (and weak) solution to problem (1.1), (1.2) as a limit of the approximate solutions $u_{\varepsilon}$ constructed in the previous section. The uniqueness in both theorems can be proved by standard considerations taking into account the monotonicity of the elliptic part of the operator (see, for example, [10]).

Let us start with the existence in Theorem 1.

Consider problem (2.2), (1.2).

From the estimates of Lemmas $2.2-2.4$ it follows that

$$
\left|u_{\varepsilon x}(t, x)-u_{\varepsilon x}(\tau, x)\right| \leq C_{2}|t-\tau|^{1 / 2}
$$

with constant $C_{2}$ depending only on $K, C_{0}$ and $C_{1}$ (see [7, Chapter II, Lemma 3.1]). Thus, taking into account inequality (3.1) and the estimates obtained in previous section we conclude that there exist a subsequence $\varepsilon_{k}$ such that

$$
u_{\varepsilon_{k}} \rightarrow u, \quad u_{\varepsilon_{k} x} \rightarrow u_{x} \quad \text { uniformly }
$$

and

$$
u_{\varepsilon_{k} t} \rightarrow u_{t}, \quad u_{\varepsilon_{k} x x} \rightarrow u_{x x}{ }^{*} \text {-weakly in } L_{\infty}\left(Q_{T}\right),
$$

as $\varepsilon_{k} \rightarrow 0$. Hence

$$
\begin{gathered}
a\left(\varepsilon_{k}, x, u_{\varepsilon_{k} x}\right) u_{\varepsilon_{k} x x} \rightarrow a\left(0, x, u_{x}\right) u_{x x}=(1+p(x))\left|u_{x}\right|^{p(x)} u_{x x}{ }^{*} \text {-weakly in } L_{\infty}\left(Q_{T}\right), \\
b\left(\varepsilon_{k}, x, u_{\varepsilon_{k} x}\right) \rightarrow b\left(0, x, u_{x}\right)=p^{\prime}(x) u_{x}\left|u_{x}\right|^{p(x)} \ln \left|u_{x}\right| \text { uniformly, } \\
f\left(x, u_{\varepsilon_{k}}, u_{\varepsilon_{k} x}\right) \rightarrow f\left(x, u, u_{x}\right) \text { uniformly. }
\end{gathered}
$$


Note that $b\left(0, x, u_{x}\right)=b\left(x, u_{x}\right)$ and recall that we put $b(x, 0)=0$. Obviously $b(\varepsilon, x, 0)=0$.

Multiplying Eq. (2.2) by an arbitrary smooth function $\phi$, integrating over $Q_{T}$ and passing to the limit when $\varepsilon_{k} \rightarrow 0$ we obtain the strong solution according to Definition 1.

Theorem 1 is proved.

Let us turn to the proof of Theorem 2 .

Consider problem (2.1), (1.2). From the estimates obtained in Lemmas 2.2 and 2.3 we have that there exists a subsequence $\varepsilon_{k}$ such that

$$
u_{\varepsilon_{k}} \rightarrow u \text { uniformly, }
$$

and

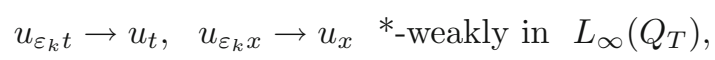

as $\varepsilon_{k} \rightarrow 0$.

Multiplying Eq. (2.1) by an arbitrary smooth function $\phi$ which vanishes on $x= \pm l$ and integrating by parts we obtain

$$
\begin{gathered}
\int_{Q_{T}} u_{\varepsilon t} \phi d x d t+\int_{Q_{T}}\left(\left|u_{\varepsilon x}\right|^{\alpha}+\varepsilon\right)^{p(x) / \alpha} u_{\varepsilon x} \phi_{x} d x d t \\
=\int_{Q_{T}}\left(g_{1}\left(x, u_{\varepsilon}\right) u_{\varepsilon x}+g_{2}\left(x, u_{\varepsilon}\right)\right) \phi d x d t .
\end{gathered}
$$

Obviously

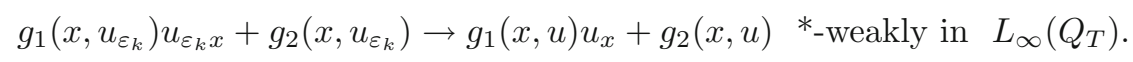

Thus, in order to pass to the limit in (3.2), we only have to prove that

$$
\int_{Q_{T}}\left(\left|u_{\varepsilon_{k} x}\right|^{\alpha}+\varepsilon_{k}\right)^{p(x) / \alpha} u_{\varepsilon_{k} x} \phi_{x} d x d t \rightarrow \int_{Q_{T}}\left|u_{x}\right|^{p(x)} u_{x} \phi_{x} d t d x \text { as } \varepsilon_{k} \rightarrow 0 .
$$

This can be done similarly to as it was done in [10](page 3018).

Theorem 2 is proved.

\section{References}

[1] Acerbi, E., Mingione, G., Seregin, G.A.: Regularity results for parabolic systems related to a class of non-Newtonian fluids. Ann. Inst. H. Poincare Anal. Non Lineaire 21(1), 25-60 (2004)

[2] Alkhutov, Yu.A., Zhikov, V.V.: Existence and uniqueness theorems for solutions of parabolic equations with a variable nonlinearity exponent (Russian). Mat. Sb. 205(3), 3-14 (2014)

[3] Antontsev, S.N., Shmarev, S.I.: Existence and uniqueness for doubly nonlinear parabolic equations with nonstandard growth conditions. Differ. Equ. Appl. 4(1), 67-94 (2012) 
[4] Antontsev, S.N., Shmarev, S.I.: Doubly degenerate parabolic equations with variable nonlinearity II: Blow-up and extinction in a finite time. Nonlinear Anal. 95, 483-498 (2014)

[5] Di Benedetto, E.: Degenerate Parabolic Equations. Springer, New York (1993)

[6] Kloeden, P., Simsen, J.: Pullback attractors for non-autonomous evolution equations with spatially variable exponents. Commun. Pure Appl. Anal. 13(6), 25432557 (2014)

[7] Ladyzhenskaja, O.A., Solonnikov, V.A., Uralceva, N.N.: Linear and quasilinear equations of parabolic type. Am. Math. Soc. Transl. (2), 23, xi+648 pp (1968)

[8] Lian, S., Gao, W., Hongjun, H., Cao, C.: Existence of solutions to an initial Dirichlet problem of evolutional $\mathrm{p}(\mathrm{x})$-Laplace equations. Ann. Inst. H. Poincare Anal. Non Lineaire 29(3), 377-399 (2012)

[9] Simsen, J., Simsen, M., Primo, M.: On $p_{s}(x)$-Laplacian parabolic problems with non-globally Lipschitz forcing term. Z. Anal. Anwend. 33(4), 447-462 (2014)

[10] Starovoitov, V.N., Tersenov, Al.: Singular and degenerate anisotropic parabolic equations with a nonlinear source. Nonlinear Anal. 72(6), 3009-3027 (2010)

[11] Tersenov, Al., Tersenov, Ar.: On the Bernstein-Nagumo's condition in the theory of nonlinear parabolic equations. J. Reine Angew. Math. 572, 197-217 (2004)

[12] Zhikov, V.V., Pastukhova, S.E.: On the property of higher integrability for parabolic systems of variable order of nonlinearity. Math. Notes 87(1-2), 169$188(2010)$

Alkis S. Tersenov

Department of Mathematics

University of Crete

71003 Heraklion, Crete

Greece

e-mail: tersenov@math.uoc.gr

Received: 23 March 2015.

Accepted: 29 February 2016. 